01

\title{
Quantum chemical insight into molecular structure, spectroscopic and nonlinear optical studies on methylene bis(dithiobenzoate)*
}

\author{
(C) Davut $\mathrm{Avci}^{1}$ and Semiha Bahceli ${ }^{2}$ \\ ${ }^{1}$ Department of Physics, Faculty of Arts and Sciences, Sakarya University, \\ 54187 Sakarya, Turkey \\ ${ }^{2}$ Emeritus Professor of Atomic and Molecular Physics, \\ Ankara, Turkey \\ e-mail: davci@sakarya.edu.tr, s.bahceli.80@gmail.com \\ Received September 29, 2020 \\ Revised May 28, 2021 \\ Accepted June 08, 2021
}

In this study, the methylene bis(dithiobenzoate) molecule, $\left(\mathrm{C}_{15} \mathrm{H}_{12} \mathrm{~S}_{4}\right)$, as a bioactive molecule has been subjected to quantum chemical computations using density functional theory (DFT) in order to investigate the molecular geometry, IR, UV-visible and NMR spectral studies. The title molecule has been optimized at the B3LYP, B3PW91 and PBE11PBE levels of DFT and 6-311G $(\mathrm{d}, \mathrm{p})$ basis set. Furthermore, the vibrational frequencies, the HOMOLUMO energy levels, the ${ }^{1} \mathrm{H}$ and ${ }^{13} \mathrm{C}$ NMR chemical shifts (ppm), nonlinear optical properties calculations of the title compound were obtained by B3LYP, B3PW91 and PBE1PBE levels. The maximum electronic transition wavelengths, oscillator strengths, excited state and transition dipole moments for the title compound were also investigated by B3LYP, B3PW91 and PBE1PBE levels of time-dependent (TD)-DFT.

Keywords: methylene bis(dithiobenzonoate), IR and UV-vis spectroscopy, ${ }^{1} \mathrm{H}$ and ${ }^{13} \mathrm{C}$ NMR chemical shifts, NLO DFT method.

* Полный текст статьи опубликован в „Optics and Spectroscopy“ 2021 V. 129. N 9. 